\title{
The Health Monitoring Method of Concrete Dams Based on Ambient Vibration Testing and Kernel Principle Analysis
}

\author{
Lin Cheng, ${ }^{1}$ Jie Yang, ${ }^{1}$ Dongjian Zheng, ${ }^{2} \mathrm{Bo} \mathrm{Li}^{3}$ and Jie Ren ${ }^{1}$ \\ ${ }^{1}$ State Key Laboratory Base of Eco-Hydraulic Engineering in Arid Area, Xian University of Technology, Xi'an 710048, China \\ ${ }^{2}$ State Key Laboratory of Hydrology-Water Resources and Hydraulic Engineering, Hohai University, Nanjing 210098, China \\ ${ }^{3}$ Engineering Safety and Disaster Prevention Department, Changjiang River Scientific Research Institute, Wuhan 430010, China
}

Correspondence should be addressed to Lin Cheng; wanmu2381@163.com

Received 21 August 2014; Accepted 28 October 2014

Academic Editor: Mickaël Lallart

Copyright (C) 2015 Lin Cheng et al. This is an open access article distributed under the Creative Commons Attribution License, which permits unrestricted use, distribution, and reproduction in any medium, provided the original work is properly cited.

\begin{abstract}
The ambient vibration testing (AVT) measurement of concrete dams on full-scale can show the practical dynamic properties of structure in the operation state. For most current researches, the AVT data is generally analyzed to identify the structural vibration characteristics, that is, modal parameters. The identified modal parameters, which can provide the global damage information or the damage location information of structure, can be used as the basis of structure health monitoring. Therefore, in this paper, the health monitoring method of concrete dams based on the AVT is studied. The kernel principle analysis (KPCA) based method is adopted to eliminate the effect of environmental variables and monitor the health of dam under varying environments. By taking full advantage of the AVT data obtained from vibration observation system of dam, the identification capabilities and the warning capabilities of structural damage can be improved. With the simulated AVT data of the numerical model of a concrete gravity dam and the measured AVT data of a practical engineering, the performance of the dam health monitoring method proposed in this paper is verified.
\end{abstract}

\section{Introduction}

The safety of concrete dams, such as gravity dams, buttress dams, and arch dams, is directly related to not only its social and economic benefits, but also the personal and property safety of residents around the reservoir area. Therefore, it is of great importance to monitor the health of concrete dams using the obtained real-time information. Structure health monitoring (SHM) [1] is a process to search for reasonable and economical ways to monitor structural state, so that its remaining structural life can be known and possibly extended. For traditional static dam health monitoring methods, the commonly measured quantities are displacement, seepage flow, temperature, and so forth. With the measured data of these quantities, we usually can only detect the local damage of a structure where the instruments are installed and it is very difficult to evaluate the global state of large hydraulic structures. The structural vibration characteristics, such as frequencies, damping ratios, and modal shapes, can reflect the local and the global structural damage information.
In addition, with the construction of vibration monitoring system of structures, such as the dam strong earthquake monitoring system and the powerhouse vibration monitoring system, the real-time vibration monitoring data of concrete dams can be acquired conveniently now. Therefore, recently, the vibration-based structural health monitoring technology is widely concerned in the hydraulic engineering [2].

For the vibration-based structure health monitoring technique, the vibration response data of a structure under external excitations should be measured by some sensors at first, and then the structural vibration characteristics are extracted using the data. The structural vibration characteristics generally refer to modal parameters, which include natural frequencies, mode shape vectors, and coordinate modal assurance criterion (COMAC). In hydraulic engineering, early dam modal identification is based on experimental modal analysis (EMA) [3] and physical model testing [4]. While the complex boundary of a dam-foundation-water system is difficult to simulate in physical model testing, the EMA suffers from certain limitations, such as a need for 
artificial vibration excitation, which is usually expensive and limited by the risk of damaging the structure. The ambient vibration testing (AVT) data of concrete dams on full-scale can show the practical dynamic properties of structure in the operation state without using any expensive artificial excitations. Therefore, it is a very good way to study the dynamic properties of dam based on the AVT and it attracted much attention in hydraulic engineering [5-11].

Although, for concrete dams and some other hydraulic structures, the good performance of vibration-based structure health monitoring method has been verified by some numerical and experimental investigations, its application in practical hydraulic engineering is still very limited. The main reason for it is that the effect of some environmental variables, such as temperature, water level, and rainfall, cannot be ignored directly in practical engineering as in numerical and experimental investigations. The environmental variability often results in changes in the structural system [12-15]. These changes, too, may be interpreted as damage, which will bring much difficulty to the health monitoring of structure. Therefore, it is very important to remove the effect of environmental variables before making heath monitoring on a dam based on the identified modal parameters. In order to make structure heath monitoring under varying environment, Sohn et al. [12], Yan et al. [13], Deraemaeker et al. [14], and Ni et al. [15] have made some research for bridge structures. For concrete dams and other hydraulic structures, since the relationship between environmental variables and structural vibration characteristics is more complex, the methods mentioned above may not be applied directly.

In order to study the health monitoring method of dam based on AVT, in this work, the modal parameters identification method of dam under ambient excitations is studied at first. With the identified modal parameters, the kernel principle analysis (KPCA) [16] based method is adopted to eliminate the effect of environmental variables. Then, the square prediction errors (SPE) control charts and the contribution plots are used to detect damage and find the locations of damage, respectively. At the final of this work, a numerical example and an engineering example are used to verify the proposed dam health monitoring method based on the AVT and KPCA.

\section{Modal Identification of Concrete Dams Using AVT}

The modal identification of concrete dams based on AVT can be categorized as the structural operation modal analysis (OMA) problem. The research on this problem attracted considerable attention recently. For example, based on the AVT, Loh and $\mathrm{Wu}$ [6] used the stochastic subspace identification (SSI) model, Kou et al. [7] used the autoregression with eXtra inputs (ARX) model, and Darbre et al. used the enhanced frequency domain decomposition (EFDD) method, respectively, to identify the modal parameters of dams. In this paper, the modal parameters identification method based on Hankel matrix joint approximate diagonalization (HJAD) technology, which has strong robustness, high computation efficiency, and being able to estimate more active modes than the number of available sensors, is adopted. The detail of HJAD-based modal identification method can be found in reference [8]. In the following section, a brief introduction of some principles of this method will be made.

2.1. The Covariance Function of Measured AVT Data. The equation of motion of an $n$ degree-of-freedom (DOF) lumped mass system can be described as [17]

$$
\mathbf{M} \ddot{\mathbf{x}}(t)+\mathbf{C} \dot{\mathbf{x}}(t)+\mathbf{K x}(t)=\mathbf{f}(t),
$$

where $\mathbf{M} \in \mathbb{R}^{n \times n}, \mathbf{C} \in \mathbb{R}^{n \times n}$, and $\mathbf{K} \in \mathbb{R}^{n \times n}$ are the mass, damping, and stiffness matrices of the system, respectively; $\mathbf{x}(t), \dot{\mathbf{x}}(t), \ddot{\mathbf{x}}(t)$, and $\mathbf{f}(t) \in \mathbb{R}^{n \times 1}$ are the relative displacement, velocity, acceleration, and force vectors; $\mathbf{f}(t)$ is generally expressed in the form $\mathbf{f}(t)=\mathbf{B}_{2} \mathbf{u}(t)$; matrix $\mathbf{B}_{2} \in \mathbb{R}^{n \times m}$ describes the $m$ inputs in space; and vector $\mathbf{u}(t) \in \mathbb{R}^{m}$ describes the $m$ inputs in time.

For a nonclassical damping system, we usually use the state space model, namely, the first order differential or difference equation of state variables, to study a structure's vibration problem. Equation (1) then can be rewritten in the state space as follows:

$$
\dot{\mathbf{z}}(t)=\mathbf{A}_{c} \mathbf{z}(t)+\mathbf{B}_{c} \mathbf{u}(t)
$$

where $\mathbf{z}(t)=\left[\mathbf{x}(t)^{T}, \dot{\mathbf{x}}(t)^{T}\right]^{T} \in \mathbb{R}^{2 n \times 1}$ is the state variable vector; the state matrix $\mathbf{A}_{c}=\left[\begin{array}{cc}\mathbf{0}_{n \times n} & \mathbf{I}_{n \times n} \\ -\mathbf{M}^{-1} \mathbf{K} & -\mathbf{M}^{-1} \mathbf{C}\end{array}\right] \in \mathbb{R}^{2 n \times 2 n} ; \mathbf{B}_{c}=$ $\left[\begin{array}{c}\mathbf{0}_{n \times 1} \\ \mathbf{M}^{-1} \mathbf{B}_{2}\end{array}\right] \in \mathbb{R}^{2 n \times m}$ is the input matrix; and the subscript $c$ indicates a continuous-time system.

The observation equation is as follows:

$$
\mathbf{y}(t)=\mathbf{G z}(t)+\mathbf{D} \mathbf{u}(t)+\mathbf{v}(t),
$$

where $\mathbf{v}(t)$ is the observation noise vector and the outputs $\mathbf{y}(t) \in \mathbb{R}^{l \times 1}$ can be the displacement, velocity, or acceleration of $l$ sensor locations. For different measurements, the corresponding observation matrix $\mathbf{G} \in \mathbb{R}^{l \times 2 n}$ and direct transfer matrix $\mathbf{D} \in \mathbb{R}^{l \times m}$ are also different. The diagonal matrices $\mathbf{C}_{d}, \mathbf{C}_{v}$, and $\mathbf{C}_{a} \in \mathbb{R}^{l \times n}$ are defined as the output matrices for displacement, velocity, and acceleration, respectively. The observation matrix can then be expressed as follows:

$$
\mathbf{G}=\left[\mathbf{C}_{d}-\mathbf{C}_{a} \mathbf{M}^{-1} \mathbf{K} \quad \mathbf{C}_{v}-\mathbf{C}_{a} \mathbf{M}^{-1} \mathbf{C}\right]
$$

For the AVT, usually the absolute acceleration responses $\ddot{\mathbf{x}}^{a}(t)$ of $l$ channels are measured. Assume the ambient excitation is in the form of support excitation; then $\mathbf{C}_{d}=\mathbf{C}_{v}=$ $\mathbf{0}_{n}, \mathbf{C}_{a}=\mathbf{I}_{l \times n}$, and $\mathbf{D}=0$. Then the observation (3) becomes

$$
\mathbf{y}(t)=\mathbf{C}_{a} \ddot{\mathbf{x}}^{a}(t)+\mathbf{v}(t)=\mathbf{G z}(t)+\mathbf{v}(t) .
$$

The discrete state space formulation of the dynamic equation as shown in (2) and (5) of an $n$ DOF system sampled at constant time intervals $T_{s}$ is given by

$$
\begin{aligned}
\mathbf{z}_{k+1} & =\mathbf{A} \mathbf{z}_{k}+\mathbf{B} \mathbf{u}_{k}, \\
\mathbf{y}_{k} & =\mathbf{G} \mathbf{z}_{k}+\mathbf{v}_{k},
\end{aligned}
$$


where $\mathbf{A}=\exp \left(\mathbf{A}_{c} T_{S}\right)$ and $\mathbf{B}=\left(\int_{0}^{T_{s}} e^{\mathbf{A}_{c} \tau} d \tau\right) \mathbf{B}_{c}$ are the state matrix and input matrix of the discrete state space model, respectively. If $\mathbf{A}_{c}$ is invertible, then $\mathbf{B}=\mathbf{A}_{c}{ }^{-1}[\mathbf{A}-\mathbf{I}] \mathbf{B}_{c}$.

The covariance function matrix $\mathbf{R}_{\mathbf{y y}}=E\left[\mathbf{y}(t) \mathbf{y}^{T}(t+\tau)\right]$ of the measured vibration response is the basis of a variety of structure modal identification methods. Therefore, it is necessary to research the mathematical expression of this matrix. Before deducing the expression of the covariance function matrix $\mathbf{R}_{\mathrm{yy}}$, some assumption should be made. As shown in Figure 1, the boundary for a dam-foundation-reservoir system is generally very complicated. Ambient excitation sources may come from an earthquake, microtremor, fluctuating pressure caused by discharge, and traffic vibrations caused by humans or vehicles or other sources. The measured vibration response of any freedom of a structure can be taken as the comprehensive effect of all these excitations. Unlike the prescribed excitation, used in numerical simulation and experiments, the ambient excitation of concrete hydraulic structures usually can not be directly measured. In practical applications, various types of ambient excitation and the observation noise are assumed to be stochastic band-limited white noise sequences.

Based on the discrete state space formulation (6) and the assumptions on ambient excitations and observation noise, the covariance function matrix can be deduced to have the expression as follows:

$$
\mathbf{R}_{\mathrm{yy}}(\tau) \approx \mathbf{G A}^{\tau} \mathbf{R}_{\mathrm{zz}}(0) \mathbf{C}^{T}, \quad\left(\tau>\tau_{0}\right) .
$$

As shown in (7), the covariance function matrix is related only to the initial state and system parameters (modal parameters) of the structure. Thus, the expression is similar to that of the structural free vibration response and the impulse response. In other words, for a structure under white noiselike support excitation, the covariance function matrix of the measured acceleration can be used to replace the structure's impulse response when the time delay is big enough; that is, $\tau>\tau_{0}$.

2.2. HJAD-Based Modal Identification Method. Define a vector $\mathbf{Y}_{k}$ which consists of the measured AVT data of $l$ channels $\mathbf{y}_{k} \in \mathbb{R}^{l \times 1}$ and its time-lagged data:

$$
\begin{array}{r}
\mathbf{Y}_{k}=\left(\begin{array}{llll}
\mathbf{y}_{k}^{T} & \mathbf{y}_{k-1}^{T} & \cdots & \mathbf{y}_{k-p+1}^{T}
\end{array}\right)^{T} \in \mathbb{R}^{2 p l \times 1}, \\
(k=1,2, \ldots, N-p+1),
\end{array}
$$

where $p$ is the time delay $(p \times l>n)$

The Hankel matrix $\mathbf{H}\left(\tau_{i}\right) \in \mathbb{R}^{2 p l \times 2 p l}$ can then be defined as follows:

$$
\begin{aligned}
& \mathbf{H}\left(\tau_{i}\right)=E\left[\mathbf{Y}(t) \mathbf{Y}^{T}\left(t+\tau_{i}\right)\right] \\
& =\left[\begin{array}{cccc}
\mathbf{R}_{\mathrm{yy}}\left(\tau_{i}\right) & \mathbf{R}_{\mathrm{yy}}\left(\tau_{i+1}\right) & \cdots & \mathbf{R}_{\mathrm{yy}}\left(\tau_{i+p-1}\right) \\
\mathbf{R}_{\mathrm{yy}}\left(\tau_{i+1}\right) & \mathbf{R}_{\mathrm{yy}}\left(\tau_{i+2}\right) & \cdots & \mathbf{R}_{\mathrm{yy}}\left(\tau_{i+p}\right) \\
\vdots & \vdots & \cdots & \vdots \\
\mathbf{R}_{\mathrm{yy}}\left(\tau_{i+p-1}\right) & \mathbf{R}_{\mathrm{yy}}\left(\tau_{i+p}\right) & \cdots & \mathbf{R}_{\mathrm{yy}}\left(\tau_{i+2 p-2}\right)
\end{array}\right], \\
& \left(\tau_{i}>\tau_{0}\right) .
\end{aligned}
$$

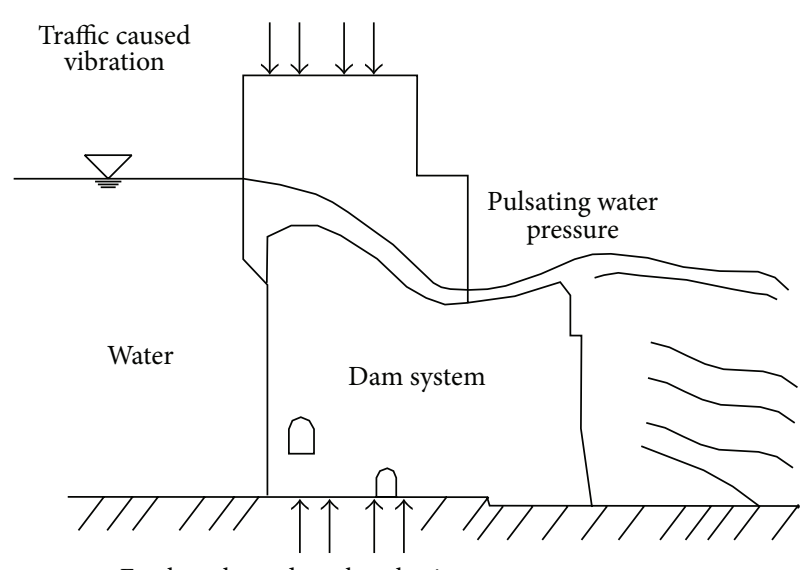

Earthquake and earth pulsation

FIgURE 1: Ambient excitation sources of dams.

As shown in (7), $\mathbf{R}_{\mathrm{yy}}\left(\tau_{j}\right)(j=i, i+1, \ldots, i+2 p-2)$ has similar expression with the structural free vibration response when the time delay $\tau_{i}$ is large enough. Based on this, the following decomposition formation of Hankel matrix $\mathbf{H}\left(\tau_{i}\right)$ can be obtained:

$$
\begin{aligned}
& \mathbf{H}\left(\tau_{i}\right)=\left[\begin{array}{c}
\boldsymbol{\Theta} \\
\boldsymbol{\Theta} \boldsymbol{\Sigma}\left(T_{s}\right) \\
\vdots \\
\boldsymbol{\Theta} \boldsymbol{\Sigma}^{p-1}\left(T_{s}\right)
\end{array}\right] \\
& \times \mathbf{R}_{\ddot{\tilde{\mathbf{q}}} \ddot{\tilde{\mathbf{q}}}}\left(\tau_{i}\right)\left[\begin{array}{llll}
\boldsymbol{\Theta}^{H} & \boldsymbol{\Sigma}\left(T_{s}\right) \boldsymbol{\Theta}^{H} & \ldots & \boldsymbol{\Sigma}^{p-1}\left(T_{s}\right) \boldsymbol{\Theta}^{H}
\end{array}\right] \\
& =\widetilde{\boldsymbol{\Theta}} \mathbf{R}_{\ddot{\mathbf{q}} \ddot{\mathbf{q}}}\left(\tau_{i}\right) \widetilde{\boldsymbol{\Theta}}^{T},
\end{aligned}
$$

where $\Theta=\left[\begin{array}{ll}\mathrm{C}_{a} \Phi^{R} & -\mathrm{C}_{a} \Phi^{I} \\ \mathrm{C}_{a} \Phi^{I} & \mathrm{C}_{a} \boldsymbol{\Phi}^{R}\end{array}\right]$ and $\boldsymbol{\Phi}^{R}$ and $\boldsymbol{\Phi}^{I}$ are the real parts and the imaginary parts of complex mode shapes, respectively; $\boldsymbol{\Sigma}\left(T_{s}\right)=\exp \left\{\boldsymbol{\Lambda} T_{s}\right\}, \boldsymbol{\Lambda}=\operatorname{diag}\left(\lambda_{1}, \ldots, \lambda_{n}\right)$, $\lambda_{1}, \ldots, \lambda_{n}$, are the eigenvalues of the system matrix $\mathbf{A}_{c}$; $\mathbf{R}_{\ddot{\mathbf{q}} \ddot{\mathbf{q}}}(\tau)=\left[\begin{array}{cc}\mathbf{R}_{\mathbf{q}^{R}{ }^{R}{ }^{R}(\tau)} & \mathbf{0} \\ \mathbf{0} & \mathbf{R}_{\mathbf{q}^{I} \dot{q}^{I}}(\tau)\end{array}\right], \mathbf{q}^{R}(t)$ and $\mathbf{q}^{I}(t)$ are the real parts and the imaginary parts of the modal response, respectively.

Then, the joint approximate diagonalization (JAD) technique can be used to realize the approximate diagonalization of the Hankel matrix for small damping structures. For a group of Hankel matrices with different timedelays $\mathbf{H}\left(\tau_{1}\right), \mathbf{H}\left(\tau_{2}\right), \ldots, \mathbf{H}\left(\tau_{T}\right)$, the generalized diagonalization matrix can be obtained by implementing joint approximate diagonalization (JAD) on the matrices, and the corresponding optimization problem is as follows:

$$
\text { minimize : } \sum_{i=1}^{T} \| \text { off }\left(\mathbf{U}^{H} \mathbf{W H}\left(\tau_{i}\right) \mathbf{W}^{H} \mathbf{U}\right) \|^{2}, \quad\left(\tau_{i}>\tau_{0}\right) .
$$

The whitening matrix $\mathbf{W}$ is obtained using the PCA method. 


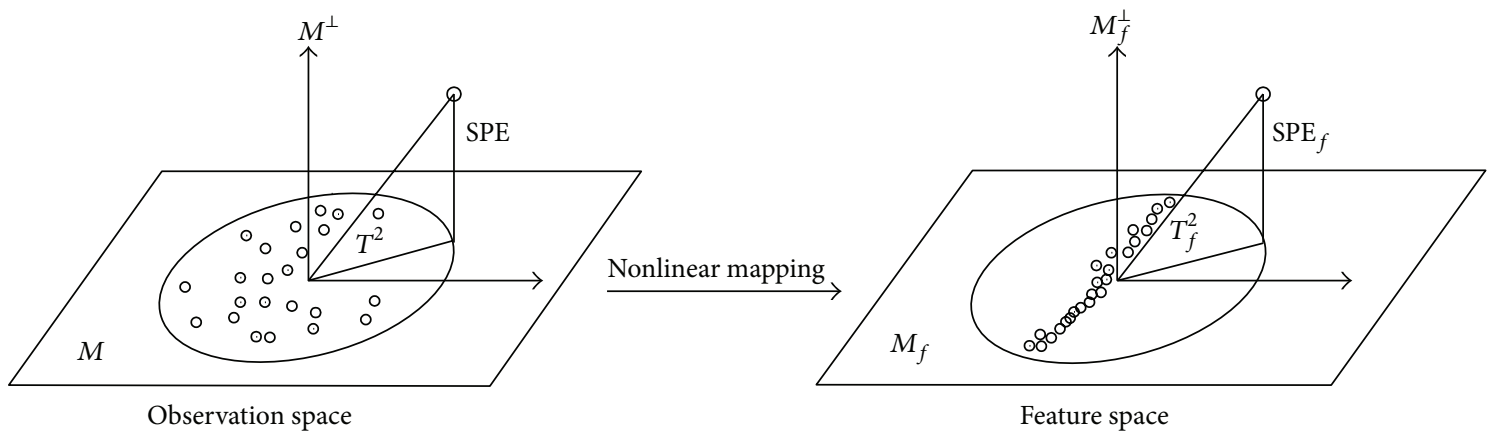

FIGURE 2: The principle of KPCA-based structure health monitoring method.

Solve the optimization problem shown above and obtain the generalized diagonalization matrix $\mathbf{U}$. Then, the separation matrix $\widetilde{\boldsymbol{\Theta}}^{+}=\mathbf{U}^{H} \mathbf{W}$ and the mixing matrix $\widetilde{\boldsymbol{\Theta}}=$ $\mathbf{W}^{+} \mathbf{U}$ can be obtained. The submatrix $\Theta=\left[\begin{array}{lll}\mathrm{C}_{a} \Phi^{R} & -\mathrm{C}_{a} \Phi^{I} \\ \mathrm{C}_{a} \Phi^{I} & \mathrm{C}_{a} \Phi^{R}\end{array}\right]$ of $\widetilde{\boldsymbol{\Theta}}$ contents the real parts $\mathbf{C}_{a} \Phi^{R}$ and the imaginary parts $\mathrm{C}_{a} \Phi^{R}$ of the observed complex mode shape. In addition, the real parts $\mathbf{q}^{R}(t)$ and the imaginary parts $\mathbf{q}^{I}(t)$ of modal response can be obtained with the separation matrix $\widetilde{\Theta}^{+}$. The modal identification method of the (single-degree-offreedom) SDOF system is then used to identify the natural frequencies and damping ratios.

The coordinate modal assurance criterion (COMAC) proposed by Lieven and Ewins [18] is calculated by

$$
\operatorname{COMAC}(k)=\frac{\sum_{r=1}^{n}\left|\Phi_{r k}^{b} \cdot \Phi_{r k}^{c}\right|^{2}}{\sum_{r=1}^{n} \Phi_{r k}^{b^{2}} \sum_{r=1}^{n} \Phi_{r k}^{c 2}}, \quad(k=1,2, \ldots, l),
$$

where $\Phi_{r k}^{b}$ an $\Phi_{r k}^{c}$ are the component $k$ of the $r$ th mode shape vector of the undamaged baseline structure and the structure to compare, respectively.

\section{Dam Health Monitoring under Varying Environment}

For dams and other hydraulic structures, the effect of some environmental variables, such as temperature, water level, and rainfall, on the identification results of vibration characteristics cannot be ignored directly in practical engineering. This may bring many difficulties to the health monitoring of dam under varying environment. In order to monitor the health of a structure under varying environment, a commonly used method which seeks to remove the variability due to environment without measuring the environmental variables is the principle analysis (PCA) method proposed by Deraemaeker et al. [14]. PCA is a linear data analysis method in nature and has good performance for processing the linear data of identified vibration characteristics. For data with strong nonlinearity, using the PCA-based structure health monitoring method will bring out great false alarm rate and missing alarm rate. Therefore, in this section, the KPCA method which is the extended nonlinear version of
PCA method is adopted to detect structural damage of dam under ambient excitation and varying environment.

3.1. The Kernel Principle Analysis Method. The learning process of PCA and KPCA needs some training samples which are vibration characteristics for the vibration-based structure health monitoring problem. As shown in Figure 2, PCA is performed in the original observation space, whereas KPCA is carried out in the extended feature space. KPCA is a type of kernel-based machine learning method in nature.

Let $\mathbf{F}$ be the identified vibration characteristics ( $n$ order natural frequencies, $l$ components of a mode shape vector, $l$ components of COMAC, etc.) and let $\mathbf{F}_{1}, \ldots, \mathbf{F}_{N}$ be the identified vibration characteristics using the observed AVT measurements of undamaged dam, at $N$ different times. The $l$ (or $n$ ) dimensional time series will be used as the training sample of KPCA learning. By a nonlinear mapping $\Theta$ : $\mathbf{F} \in \mathbb{R}^{l} \rightarrow \boldsymbol{\xi} \in \mathbb{R}^{h}$, the samples are extended into the hyperdimensional feature space. The dimension of feature space, $h$, can be arbitrarily large or even infinite. After using the nonlinear mapping, the data in the feature space may have more simple structure than in the original observation space.

Assuming $\Theta\left(\mathbf{F}_{1}\right), \ldots, \Theta\left(\mathbf{F}_{N}\right)$ have been mean-centered, then the linear PCA can be conducted in the extended dimensional feature space by diagonalizing the sample covariance matrix $\mathbf{S} \in \mathbb{R}^{h \times h}$ which can be calculated in the feature space as follows:

$$
\mathbf{S}=\frac{1}{N} \sum_{i=1}^{N} \Theta\left(\mathbf{F}_{i}\right) \Theta\left(\mathbf{F}_{i}\right)^{T}
$$

In order to perform PCA, the eigenvalue decomposition is implemented to the matrix $\mathbf{S}$, that is, solving the following eigenvalue problem:

$$
\beta \mathbf{v}=\mathbf{S v}=\frac{1}{N} \sum_{i=1}^{N}\left\langle\Theta\left(\mathbf{F}_{i}\right)^{T} \mathbf{v}\right\rangle \Theta\left(\mathbf{F}_{i}\right),
$$

where $\beta>0$ is the eigenvalue and $\mathbf{v} \neq \mathbf{0}$ is the eigenvectors of the sample covariance matrix $\mathbf{S}$. All the solutions $\mathbf{v}$ lie in the 
span $\left(\Theta\left(\mathbf{F}_{1}\right), \ldots, \Theta\left(\mathbf{F}_{N}\right)\right)$ and can be expressed by the linear combination of $\Theta\left(\mathbf{F}_{1}\right), \ldots, \Theta\left(\mathbf{F}_{N}\right)$ as

$$
\mathbf{v}=\sum_{i=1}^{N} \alpha_{i} \Theta\left(\mathbf{F}_{i}\right)
$$

The problem is then reduced to that of finding the coefficients $\alpha_{i}(i=1,2, \ldots, N)$.

Multiply with $\Theta\left(\mathbf{F}_{j}\right)$ from the left of both sides in (14), then the following expression can be obtained:

$$
\beta\left(\Theta\left(\mathbf{F}_{j}\right) \cdot \mathbf{v}\right)=\Theta\left(\mathbf{F}_{j}\right) \cdot \mathbf{S v} .
$$

By defining the kernel matrix $K_{i j}=\left\langle\Theta\left(\mathbf{F}_{i}\right), \Theta\left(\mathbf{F}_{j}\right)\right\rangle,(i, j=$ $1,2, \ldots, N)$, the eigenvalue problem shown in (14) can be rewritten as

$$
\beta \boldsymbol{\alpha}=\left(\frac{1}{N}\right) \mathbf{K} \boldsymbol{\alpha}
$$

where $\boldsymbol{\alpha} \in \mathbb{R}^{N},\|\boldsymbol{\alpha}\|^{2}=1 / N \beta$.

The kernel matrix $(\mathbf{K})_{i j}=\kappa\left(\mathbf{x}_{i}, \mathbf{x}_{j}\right)=\Theta\left(\mathbf{x}_{i}\right)^{T} \Theta\left(\mathbf{x}_{j}\right)$ should satisfy Mercer's condition such that $(\mathbf{K})_{i j}$ corresponds to an inner product in the feature space. The kernel matrix is calculated using the kernel function $\kappa(\cdot)$. The most commonly used kernel function is the Gaussian kernel function $\kappa\left(\mathbf{F}_{i}, \mathbf{F}_{j}\right)=$ $\exp \left(-\left|\mathbf{F}_{i}-\mathbf{F}_{j}\right| / \sigma^{2}\right)$. The parameter $\sigma$ is automatically determined to maximize the information (variance) of the first principal component, since it is relevant to the operational variation. To accomplish this, the width value that maximizes the difference between the first and second eigenvalues is selected.

After constructing the principal components in the feature space, the $k$ th projection of the centered value $\Theta\left(\mathbf{F}_{i}\right)$ in feature space of the new sample $\mathbf{F}_{\text {new }}$ is calculated using the following equation:

$$
\begin{array}{r}
t_{\mathrm{new}, k}=\left(\mathbf{v}_{k} \cdot \Theta\left(\mathbf{F}_{\mathrm{new}}\right)\right)=\sum_{i=1}^{N} \alpha_{i}^{k} \Theta\left(\mathbf{F}_{j}\right) \cdot \Theta\left(\mathbf{F}_{\mathrm{new}}\right), \\
(k=1,2, \ldots, d),
\end{array}
$$

where $d$ is the number of principle components in the feature space.

After calculating the nonlinear principles of identified vibration characteristics based on the KPCA, the reconstructed data $\widetilde{\mathbf{F}}$ in the original observation space, which reflects the effect of environmental variables, can be calculated using the method proposed by Mika et al. [16].

Then the SPE metrics can be calculated by

$$
\mathrm{SPE}=\|\mathbf{F}-\widetilde{\mathbf{F}}\|^{2}
$$

where $\|\cdot\|^{2}$ is the $L_{2}$ norm operator.

For a structure without damage, the error vector $\boldsymbol{\varepsilon}=\mathbf{F}-\widetilde{\mathbf{F}}$ is mainly the effect of noise and other stochastic distribution. If the error vector $\boldsymbol{\varepsilon}$ is assumed to be normally distributed, the upper control limit (UCL) of SPE can be determined using the following equation [19]:

$$
\mathrm{UCL}=\theta_{1}\left[\frac{c_{\alpha} \sqrt{2 \theta_{2} h_{0}^{2}}}{\theta_{1}}+1+\frac{\theta_{2} h_{0}\left(h_{0}-1\right)}{\theta_{1}^{2}}\right]^{1 / h_{0}}
$$

where $\theta_{j}=\sum_{i=m+1}^{l} \gamma_{i}^{j}, \gamma_{i}$ the $i$ th eigenvalue of the covariance matrix of samples; $h_{0}=1-2 \theta_{1} \theta_{3} / 3 \theta_{2}^{2} ; c_{\alpha}$ is the critical value of normal distribution when the testing level is $\alpha$.

For a structure with damage, the error vector $\boldsymbol{\varepsilon}$ will include the effect of structure damage; then the distribution of it will change and the calculated SPE metrics will exceed the UCL and then the damage of structure can be found.

3.2. The Contribution Plots. When the calculated SPE norm exceeds the UCL, the contribution plots [20] can be adopted to find the locations of structural damage. Rewrite the expression of SPE shown in (19) as follows:

$$
\mathrm{SPE}=\sum_{j=1}^{l}\left\|\mathbf{F}^{j}-\widetilde{\mathbf{F}}^{j}\right\|^{2}=\sum_{j=1}^{l} \operatorname{Cspe}_{j}
$$

in which $\mathbf{F}^{j}$ is a vector which is composed of the $j$ th component of the identified modal parameters; $\widetilde{\mathbf{F}}^{j}$ is a vector composed of the reconstructed data of $\mathbf{F}^{j}$ by KPCA; Cspe ${ }_{j}$ is the contribution of component $j$ of vibration characteristic to the SPE norm.

For modal shapes, COMAC, and other metrics which can give the information of damage location, if the contribution of its $j$ th component to the SPE norm is obviously larger than that of other components, it means that damage may occur around the locations corresponding to the component $j$. Therefore, using the SPE contribution plots and modal parameters with damage location information, it is easily to find structure damage locations.

3.3. The Procedures of Dam Health Monitoring Based on KPCA. Based on the analysis above, some main steps of dam health monitoring method based on AVT and KPCA are summarized as follows (see Figure 3).

(1) For a dam in normal state, obtain the AVT data of it at $N$ different times and perform processes (removing the trend, denoising, etc.) to the data.

(2) Use the HJAD-based method to identify the $n$ modes of dam based on the processed AVT record. Then, we can obtain the time series $\mathbf{F}_{1}, \ldots, \mathbf{F}_{N}$ of identified modal parameters with the sample number equal to $N$.

(3) After normalizing these data of modal parameters by its mean $\mu$ and variation $\sigma$, the KPCA method is adopted to analyze the normalized multidimensional time series $\mathbf{F}_{1}, \ldots, \mathbf{F}_{N}$. Using the reconstructed data $\widetilde{\mathbf{F}}$, the SPE metrics are calculated using (19). Given a testing level $\alpha$, the UCL of SPE is determined by (20) for the dam without damage. 
TABLE 1: The comparison of false alarm rate and missing alarm rate of PCA and KPCA method.

\begin{tabular}{|c|c|c|c|c|c|}
\hline \multirow{3}{*}{ Case } & \multirow{3}{*}{ Indicator } & \multicolumn{4}{|c|}{ Analysis method } \\
\hline & & \multicolumn{2}{|l|}{ PCA } & \multicolumn{2}{|l|}{ KPCA } \\
\hline & & False (missing) alarm rate & Accuracy & False (missing) alarm rate & Accuracy \\
\hline \multirow{2}{*}{ Case 2 (undamaged) } & Frequency & $11.11 \%$ & $88.89 \%$ & $2.78 \%$ & $97.22 \%$ \\
\hline & COMAC & $2.78 \%$ & $97.22 \%$ & $5.56 \%$ & $94.44 \%$ \\
\hline \multirow{2}{*}{ Case 3 (damaged) } & Frequency & $80.55 \%$ & $19.44 \%$ & $5.56 \%$ & $94.44 \%$ \\
\hline & COMAC & $50.00 \%$ & $50.00 \%$ & $2.78 \%$ & $97.22 \%$ \\
\hline \multirow{2}{*}{ Case 4 (damaged) } & Frequency & $0.00 \%$ & $100.00 \%$ & $0.00 \%$ & $100.00 \%$ \\
\hline & COMAC & $11.11 \%$ & $88.89 \%$ & $0.00 \%$ & $100.00 \%$ \\
\hline
\end{tabular}

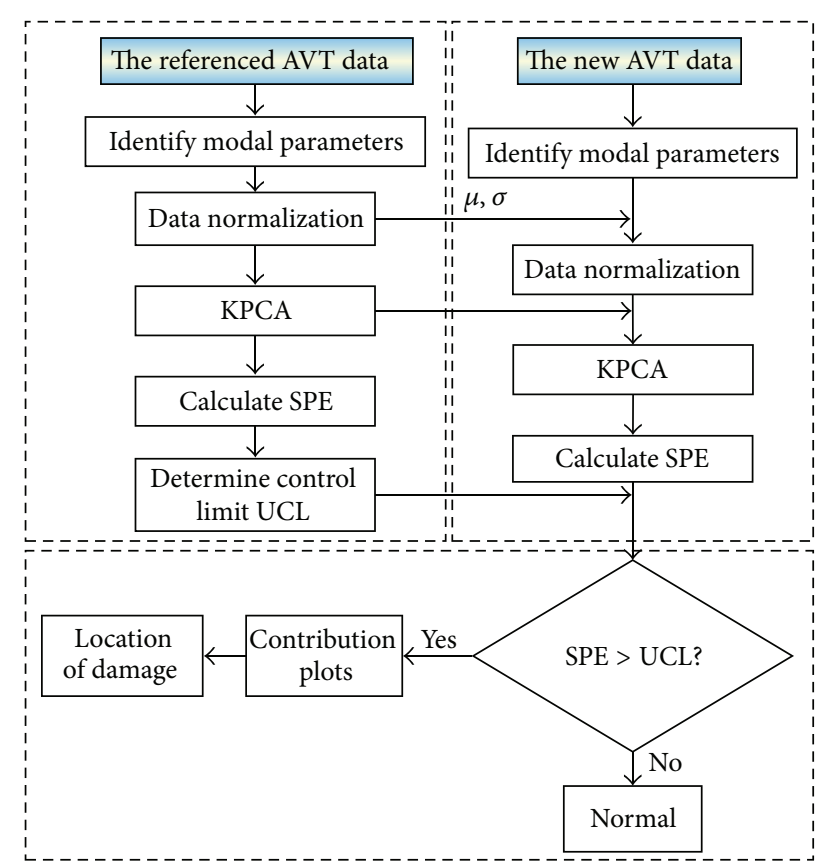

FIGURE 3: The dam health monitoring method based on AVT and KPCA.

(4) When the health state of dam is needed to be diagnosed, the new AVT is obtained and the steps 1 step 3 shown above are repeated. The new SPE is calculated and compared with the control limits calculated using (20). If the new SPE metrics exceed the control limit, the dam is abnormal; otherwise, it is still in normal state.

(5) If the dam is abnormal, for modal shapes, COMAC, and other metrics which can indict structure damage locations, the contribution plots of SPE are used to determine the location of damage. For the contribution plots, if the contribution of component $j$ to the SPE norm is obviously larger than other components, it means that damage may occur around the locations corresponding to the component $j$.

\section{Case Study}

4.1. Numerical Verification. The maximum dam block of a concrete gravity dam is used as example to verify the proposed structure health monitoring method based on AVT and KPCA method. The finite element method (FEM) is used to obtain the simulated AVT data of dam with different damage extent. The size and the finite element model of the dam block are shown in Figure 4. The elastic modulus, Poisson's ratio, and mass density of dam concrete are $31.0 \mathrm{Gpa}, 0.2$, and $2643 \mathrm{~kg} / \mathrm{m}^{3}$, respectively; the elastic modulus and Poisson's ratio of foundation rock are $20.0 \mathrm{Gpa}$ and 0.25 , respectively. The no-mass-spring is used to model the foundation when calculating the vibration response of structure. A crack near the dam heel is used to simulate the structure damage. The AVT data is obtained by adding the calculation results of the FEM software MSC.Marc with some noise with the signal-noise-ratio (SNR) equal to $50 \mathrm{db}$.

In order to evaluate the impact of environmental variables (here only the water level is simulated) on the identification results of vibration characteristics, four cases are designed. Case 1: the dam is undamaged and the water level varies between $70 \mathrm{~m}$ and $95 \mathrm{~m}$. Case 2: the dam is undamaged and the water level varies between $70 \mathrm{~m}$ and $95 \mathrm{~m}$ which are different with case 1. Case 3: the dam is damaged, the crack length is $8 \mathrm{~m}$ and the water level varies between $70 \mathrm{~m}$ and $95 \mathrm{~m}$. Case 4: the dam is damaged, the crack length is $16 \mathrm{~m}$ and the water level varies between $70 \mathrm{~m}$ and $95 \mathrm{~m}$. For each of the four cases shown above, 36 different water levels are selected. Then the vibration responses in stream direction corresponding to instruments numbered $1 \# \sim 5 \#$ are calculated using the FEM and the modal parameters of structure are identified using the simulated AVT data and HJAD-based method.

The identified modal parameters using the simulated AVT data of case 1 are selected as referenced data. Then the health state of dam corresponding to other three cases, that is, case 2 case 4 , is diagnosed using the PCA and KPCAbased method. For the referenced data, the UCL of SPE norm is calculated by the testing level $\alpha=0.001$. Based on the computation results of COMAC, the dam health monitoring results using the PCA and KPCA-based method are shown in Figures 5 and 6, respectively.

The comparison of false alarm rate and missing alarm rate between PCA and KPCA method are shown in Table 1. The false alarm rate is defined as the percentage of alarming times to all the observation times for the undamaged dam; the missing alarm rate is defined as the percentage of missing alarm times to all the observation times for the dam with 


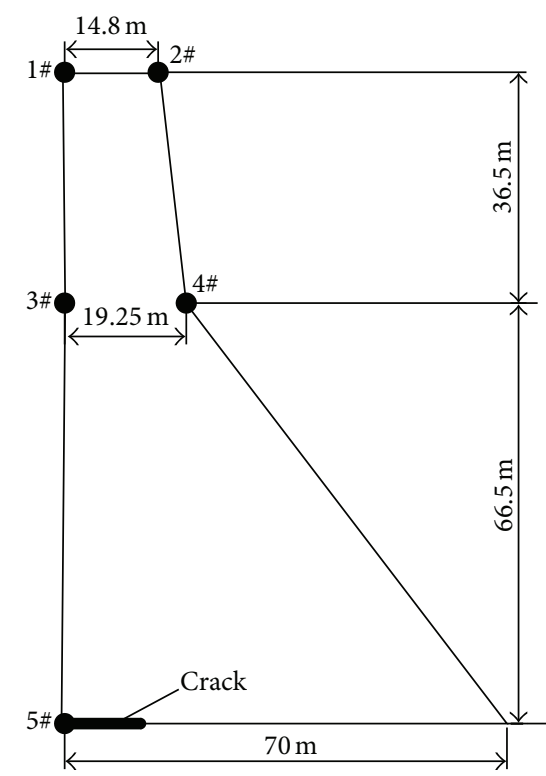

(a)

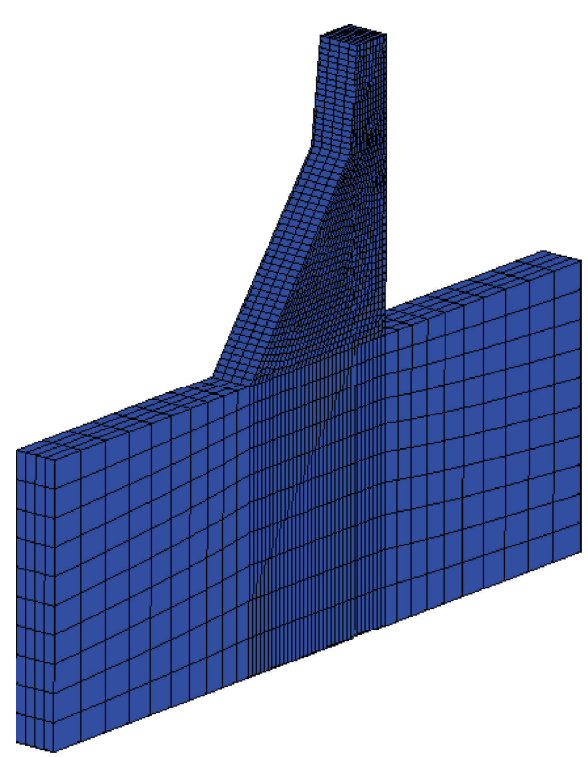

(b)

FIgURE 4: The numerical model, (a) the model size, and (b) the finite element model.

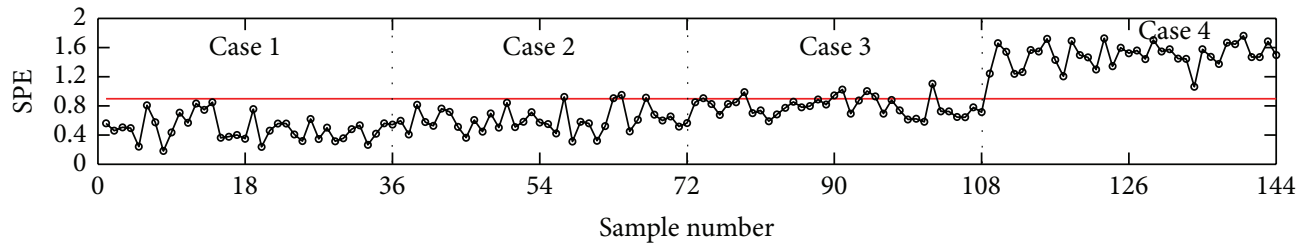

(a)

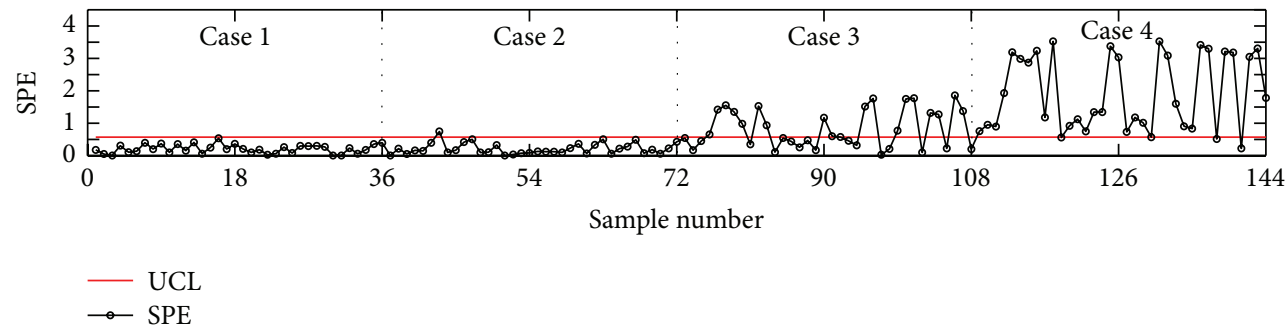

(b)

FIGURE 5: Monitoring charts of PCA-based method using (a) natural frequencies and (b) COMAC.

damage. From Figures 5 and 6 and Table 1, it can be seen that the KPCA-based dam health monitoring method can reduce the miss alarm rate and false alarm rate considerably, especially when the structure damage is small.

The SPE contribution plots of COMAC for three cases are shown in Figure 7. From this figure, it can be seen that, for case 2, the SPE contribution has no obvious trend. But for the case 3 and case 4 , the contribution of the instrument $5 \#$ is obviously bigger than that of other measurement points. This means that the structure damage is around the instrument $5 \#$, which is in accordance with the practical location of simulated crack.
4.2. Analyze the Filed Testing Data. A hydropower station is located in the middle stream of the Minjiang River in the Fujian province of China. The project is composed mainly of four parts: a roller-compacted concrete (RCC) gravity dam, a ship lock, a ship elevator, and a power generation system. The maximum height of the RCC gravity dam is $101.0 \mathrm{~m}$ and its normal flood level is $65.0 \mathrm{~m}$, with a corresponding storage capacity of $2.6 \times 10^{9} \mathrm{~m}^{3}$. This project is located near the Taiwan Strait seismic zone, so seismographs are installed on the 19th (Table 2) and 25th dam blocks. The arrangement and location of these seismographs are shown in Figure 8. In this study, only the vibration response record of dam block 19 


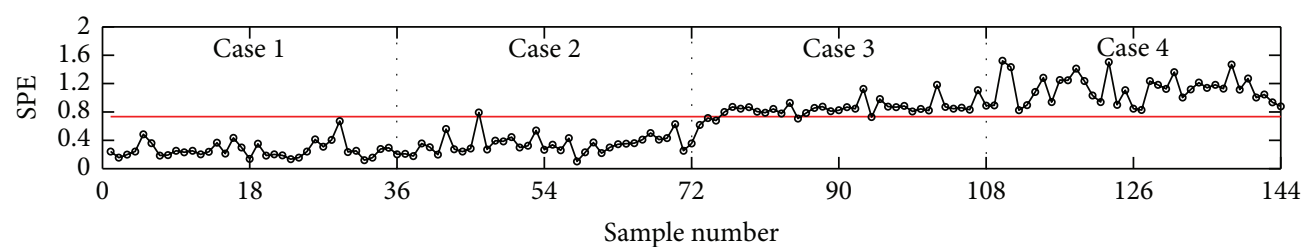

(a)

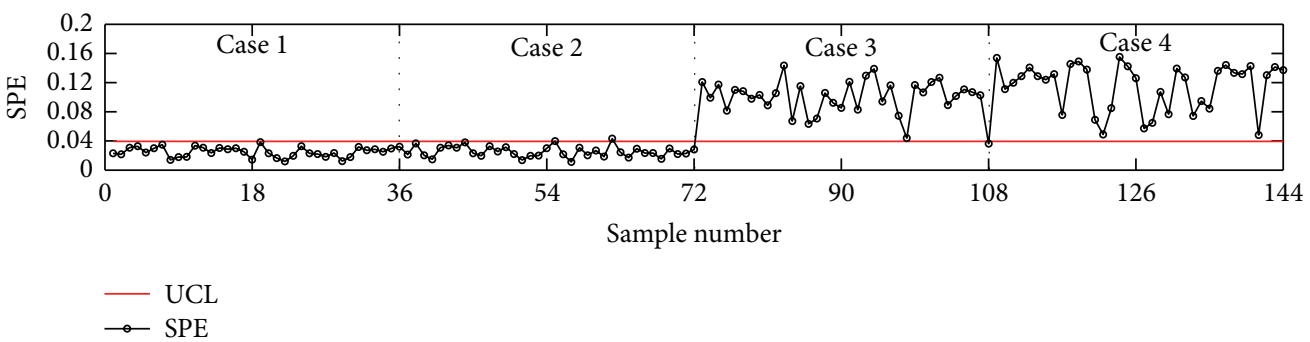

(b)

FIGURE 6: Monitoring charts of KPCA-based method using (a) natural frequencies and (b) COMAC.

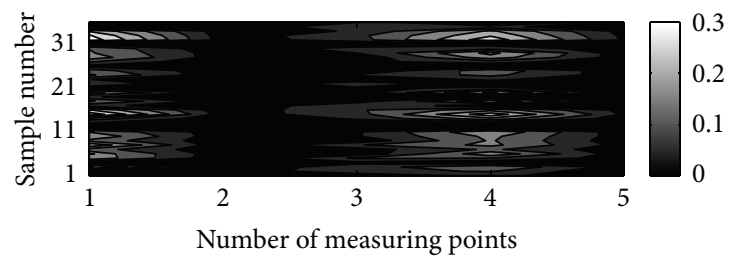

(a)

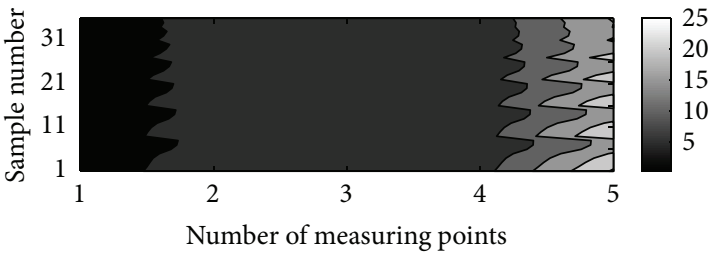

(b)

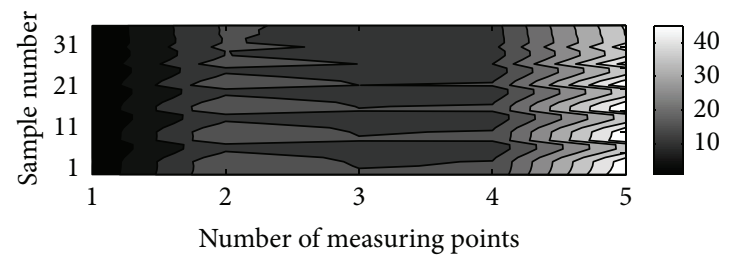

(c)

FIGURE 7: The SPE contribution plots of (a) case 2, (b) case 3, and (c) case 4.

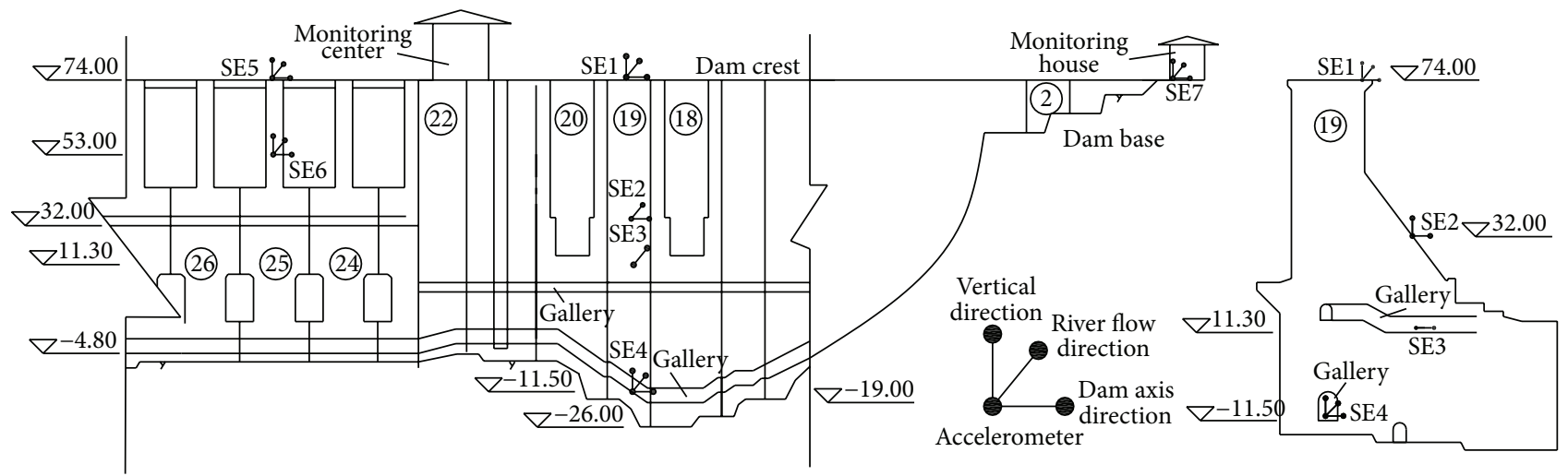

FIGURE 8: Arrangement of seismographs. 
TABLE 2: Seismographs installed on dam block number 19.

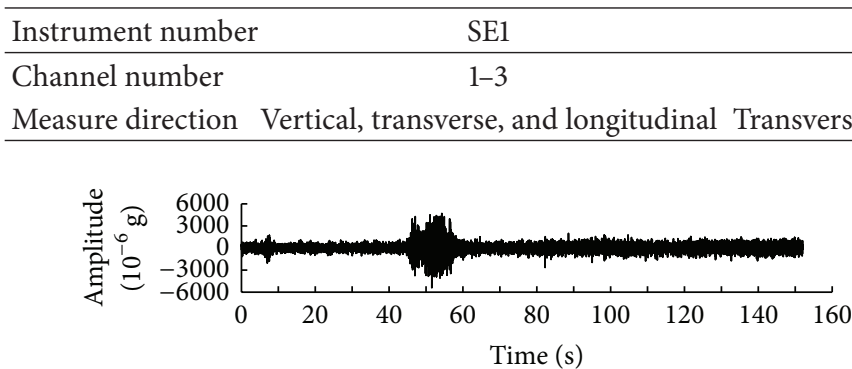

(a)

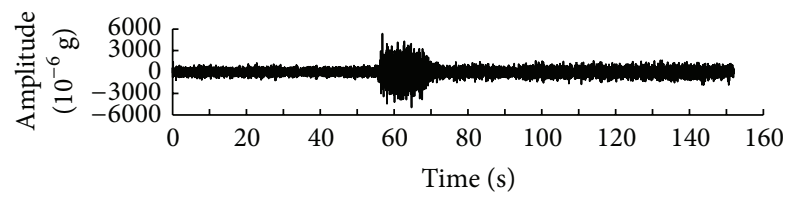

(c)

$\begin{array}{ccc}\text { SE2 } & \text { SE3 } & \text { SE4 } \\ 4-5 & 6 & 7-9\end{array}$

and longitudinal Longitudinal Vertical, transverse, and longitudinal

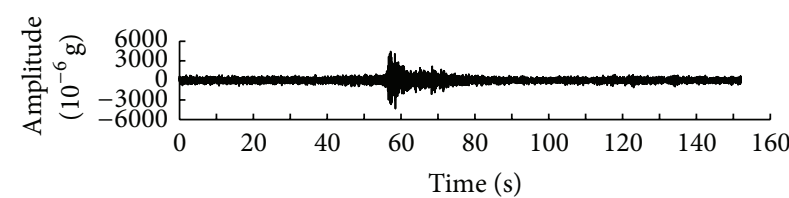

(b)

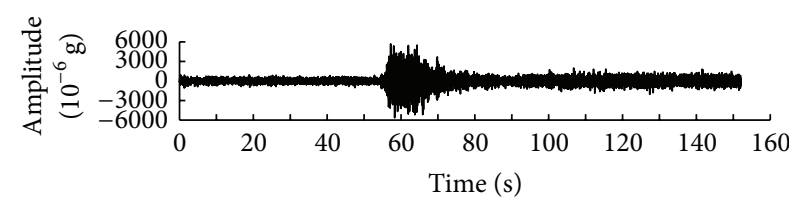

(d)

FIgURE 9: The response of channel 3 (unit: $10^{-6} \mathrm{~g}$ ).

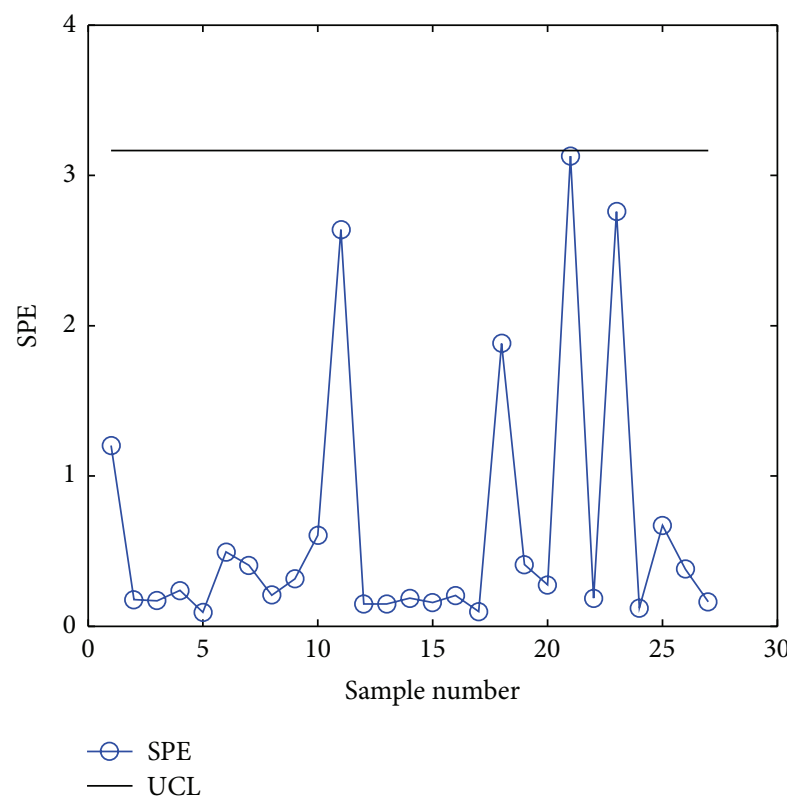

(a) Reference data

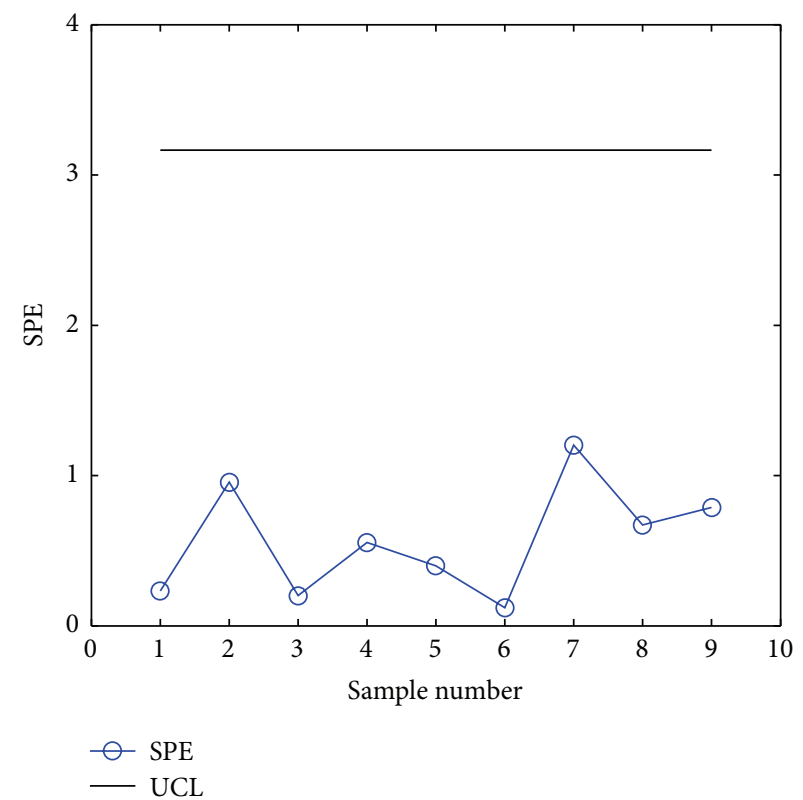

(b) Monitored

FIGURE 10: Monitoring charts of KPCA using natural frequencies.

is studied. The vibration record of 36 different days is used. The sample size of each earthquake record is 12,000 and the sampling frequency is $100 \mathrm{~Hz}$. The seismic response record of channel 3 in four different days is shown Figure 9.

The finite element software MS.Marc is used to calculate the structure's natural frequencies. A comparison between the identified frequencies using the HJAD-based method and the natural frequencies calculated using finite element method (FEM) is shown in Table 3. From Table 3, we can see that some modes cannot be identified. This may be because the modal responses of these modes are too weak to identify.
There are some differences between the calculated and the identified natural frequencies, which may be caused by the simulation errors of FEM.

The identified natural frequencies and COMAC of 5 orders using the AVT data of dam at 27 different times are used as reference data. The identified natural frequencies and COMAC at other 9 different times are used as monitored data. The dam health diagnosis result based on these data using the KPCA method is shown in Figures 10 and 11. UCL is calculated by setting the test level $\alpha=0.001$ using (20). From the two figures, we can see that the health state of 


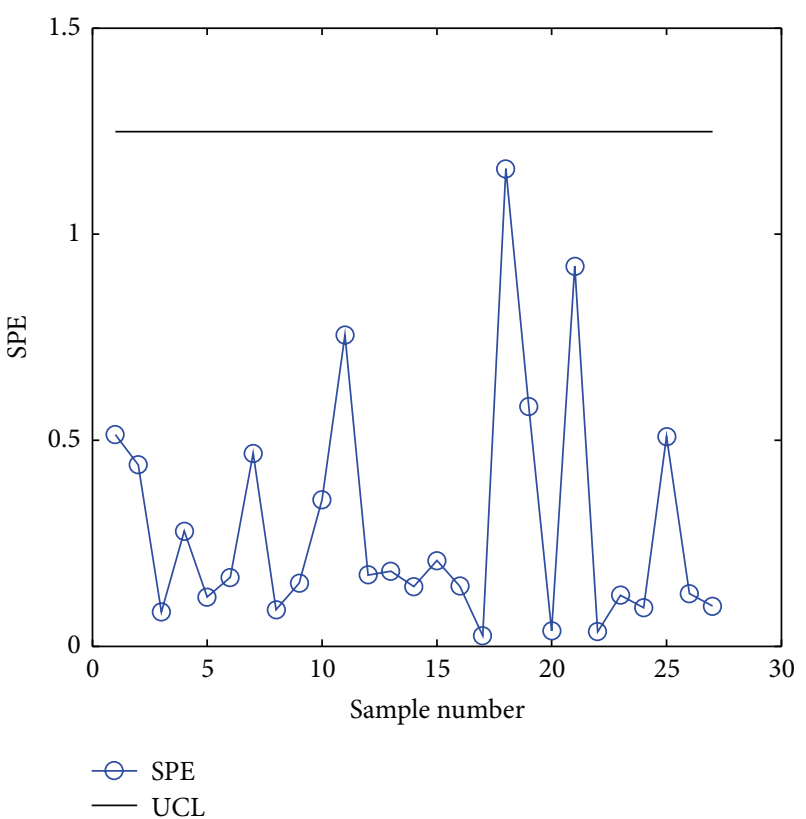

(a) Reference data

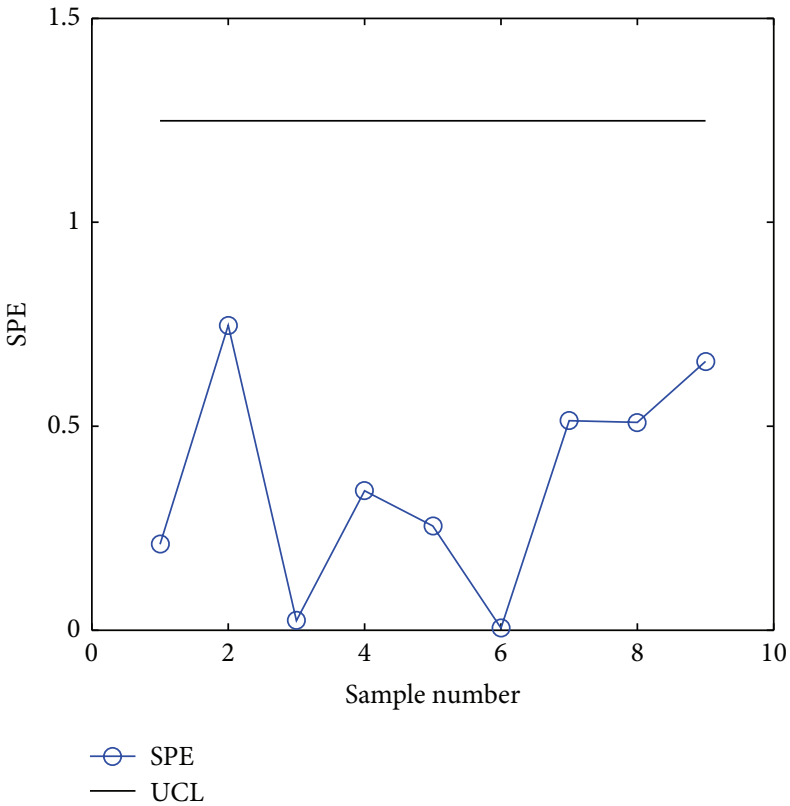

(b) Monitored

FIgURE 11: Monitoring charts of KPCA using COMAC.

TABLE 3: The identified natural frequencies of dam.

\begin{tabular}{lcccccccccc}
\hline Mode orders & 1 & 2 & 3 & 4 & 5 & 6 & 7 & 8 & 9 & 10 \\
\hline FEM & 1.01 & 3.24 & 3.84 & 4.38 & 7.43 & 7.87 & 8.98 & 9.03 & 10.99 & 12.45 \\
HJAD & 1.05 & 3.14 & - & 4.22 & 7.70 & - & 8.74 & 9.10 & 10.76 & 12.29 \\
\hline
\end{tabular}

dam is normal at the 9 different times, which is consistent with the diagnosis result using the static monitoring data of displacement and flow seepage.

\section{Conclusions}

The analysis results of the numerical example and the engineering example show that the dam health monitoring method based on AVT and KPCA is reasonable and effective. Compared to the traditional PCA-based method, the KPCAbased dam health monitoring method can improve the accuracy of alarm rate considerably. Using some modal metrics which can give the information of damage location and the contribution plots, the damage location of structure can be found. With the extensive application vibration monitoring system of dam, such as earthquake observation system, it becomes more and more easily to obtain the AVT data of dam. Thus, the dam health monitoring method proposed in this work has good prospect to be applied to more practical engineering and it has significant meaning to make further research on this problem.

\section{Conflict of Interests}

The authors declare that there is no conflict of interests regarding the publication of this paper.

\section{Acknowledgments}

This work was supported by the National Natural Science Foundation of China (Grant nos. 51409205, 51279052, 51409018, and 51309189) and the Program 2013KCT-15 for Shanxi Provincial Innovative Research Team.

\section{References}

[1] S. W. Doebling, C. R. Farrar, and M. B. Prime, Damage Identification and Health Monitoring of Structural and Mechanical Systems from Changes in Their Vibration Characteristics: A Literature Review, Alamos National Laboratory, Los Alamos, NM, USA, 1996.

[2] H. Sohn, C. R. Farrar, and F. M. Hemez, "A review of structural health monitoring literature: 1996-2001," Los Alamos National Laboratory Report LA-13976-MS, 2006.

[3] G. Zhang, C. Zhang, W. Li, and G. Wang, "Vibration measurement and analysis of Quanshui arch dam," Science China, Series A, vol. 1, pp. 100-112, 1986.

[4] G. R. Darbre, C. A. M. de Smet, and C. Kraemer, "Natural frequencies measured from ambient vibration response of the arch dam of Mauvoisin," Earthquake Engineering and Structure Dynamics, vol. 29, no. 5, pp. 577-586, 2000.

[5] J. Lian, H. Li, and J. Zhang, "ERA modal identification method for hydraulic structures based on order determination and noise reduction of singular entropy," Science in China Series E: Technological Sciences, vol. 52, no. 2, pp. 400-412, 2009.

[6] C.-H. Loh and T.-C. Wu, "System identification of Fei-Tsui arch dam from forced vibration and seismic response data," Journal of Earthquake Engineering, vol. 4, no. 4, pp. 511-537, 2000.

[7] L. Kou, F. Jin, J. Yang, and J. Wang, "Modal parameter identification of Ertan arch dam from strong earthquake records," Journal of Hydroelectric Engineering, vol. 28, no. 5, pp. 51-56, 2009. 
[8] L. Cheng and D. Zheng, “The identification of a dam's modal parameters under random support excitation based on the Hankel matrix joint approximate diagonalization technique," Mechanical Systems and Signal Processing, vol. 42, no. 1-2, pp. 42-57, 2014.

[9] C.-H. Loh and T.-S. Wu, "Identification of Fei-Tsui arch dam from both ambient and seismic response data," Soil Dynamics and Earthquake Engineering, vol. 15, no. 7, pp. 465-483, 1996.

[10] S. T. Mau and S. Wang, "Arch dam system identification using vibration test data," Earthquake Engineering \& Structural Dynamics, vol. 18, no. 4, pp. 491-505, 1989.

[11] B. Sevim, A. C. Altunişik, and A. Bayraktar, "Earthquake behavior of berke arch dam using ambient vibration test results," Journal of Performance of Constructed Facilities, vol. 26, no. 6, pp. 780-792, 2012.

[12] H. Sohn, M. Dzwonczyk, and E. G. Straser, "An experimental study of temperature effect on modal parameters of the Alamos Canyon bridge," Earthquake Engineering and Structural Dynamics, vol. 28, no. 8, pp. 879-897, 1999.

[13] A.-M. Yan, G. Kerschen, P. de Boe, and J.-C. Golinval, "Structural damage diagnosis under varying environmental conditions-part I: a linear analysis," Mechanical Systems and Signal Processing, vol. 19, no. 4, pp. 847-864, 2005.

[14] A. Deraemaeker, E. Reynders, G. De Roeck, and J. Kullaa, "Vibration-based structural health monitoring using outputonly measurements under changing environment," Mechanical Systems and Signal Processing, vol. 22, no. 1, pp. 34-56, 2008.

[15] Y. Q. Ni, X. G. Hua, K. Q. Fan, and J. M. Ko, "Correlating modal properties with temperature using long-term monitoring data and support vector machine technique," Engineering Structures, vol. 27, no. 12, pp. 1762-1773, 2005.

[16] S. Mika, B. Schölkopf, and A. S. K. Müller, "Kernel PCA and denoising in feature spaces," in Proceedings of the Conference on Advances in Neural Information Processing Systems II, pp. 536542, Cambridge, UK, 1998.

[17] B. Peeters and G. De Roeck, "Stochastic system identification for operational modal analysis: a review," Journal of Dynamic Systems, Measurement and Control, Transactions of the ASME, vol. 123, no. 4, pp. 659-667, 2001.

[18] N. A. J. Lieven and D. J. Ewins, "Spatial correlation of mode shapes, the coordinate modal assurance criterion," in Proceedings of the 6th International Modal Analysis Conference, vol. 1, pp. 690-695, Kissimmee, Fla, USA, 1988.

[19] S. W. Choi, C. Lee, J.-M. Lee, J. H. Park, and I.-B. Lee, "Fault detection and identification of nonlinear processes based on kernel PCA," Chemometrics and Intelligent Laboratory Systems, vol. 75, no. 1, pp. 55-67, 2005.

[20] P. Miller, R. E. Swanson, and C. E. Heckler, "Contribution plots: a missing link in multivariate quality control," Applied Mathematics and Computer Science, vol. 8, no. 4, pp. 775-792, 1998. 

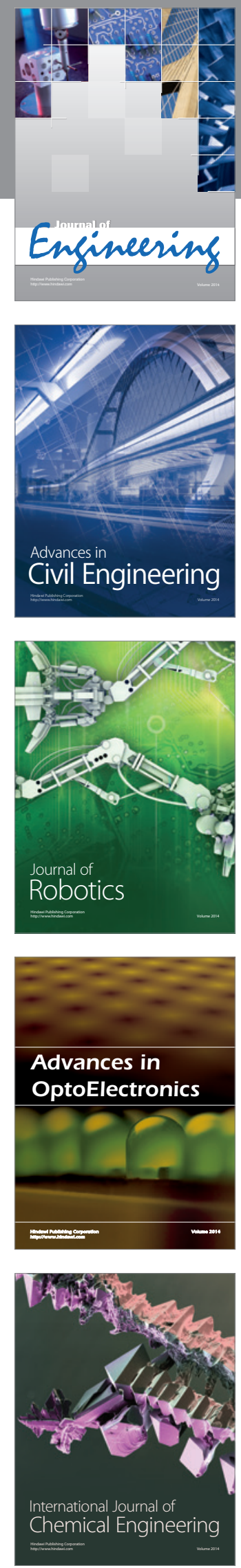

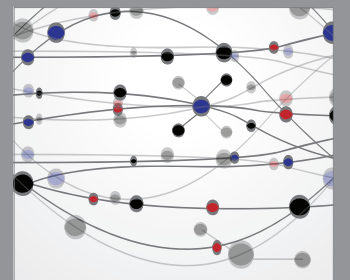

The Scientific World Journal
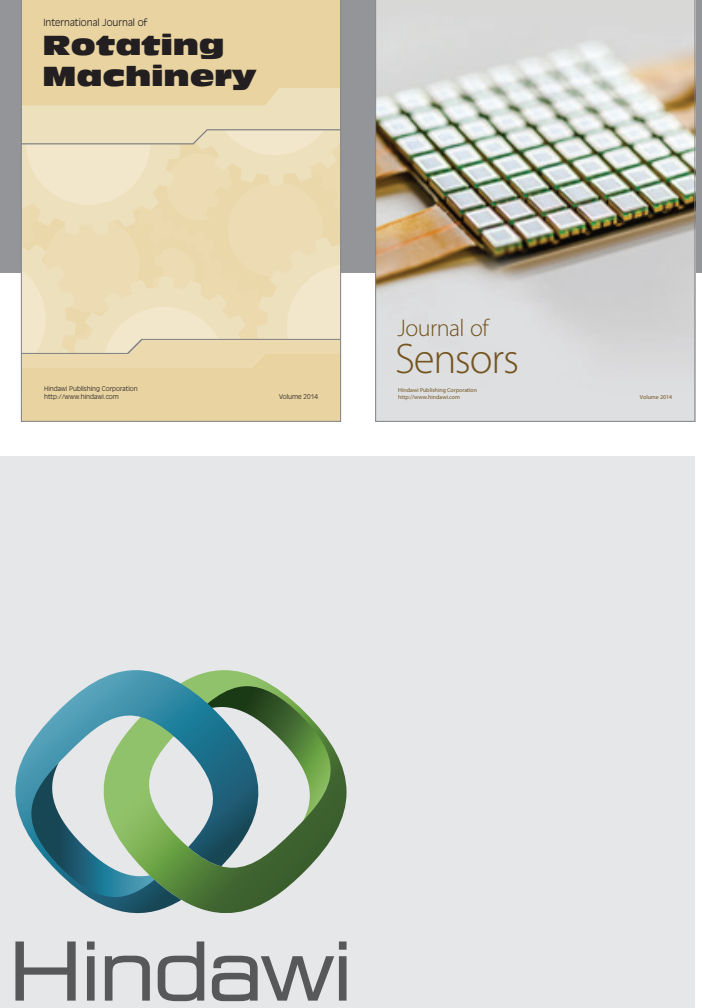

Submit your manuscripts at http://www.hindawi.com
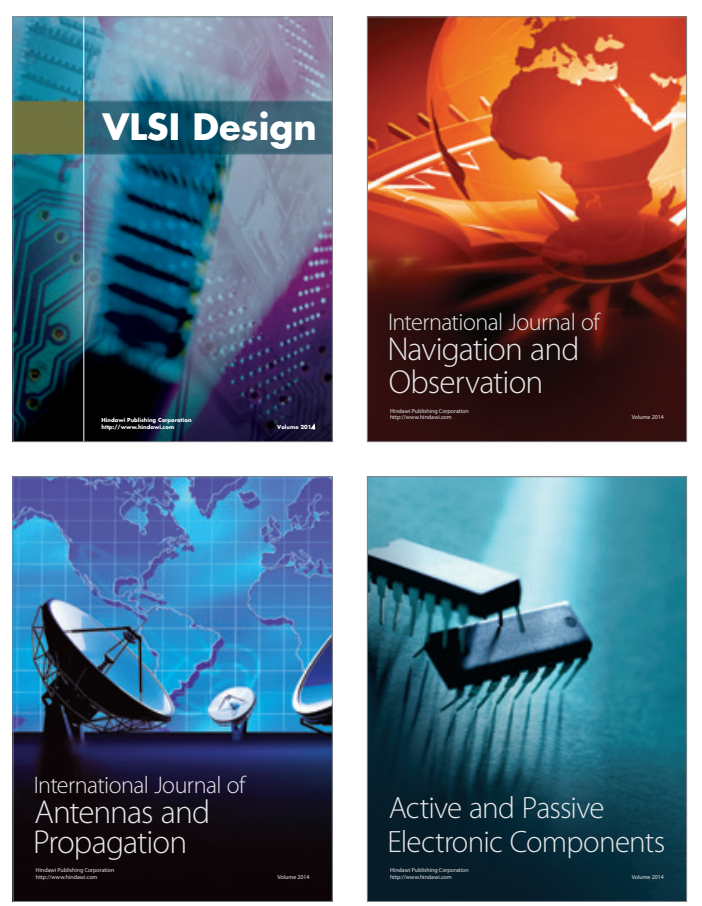
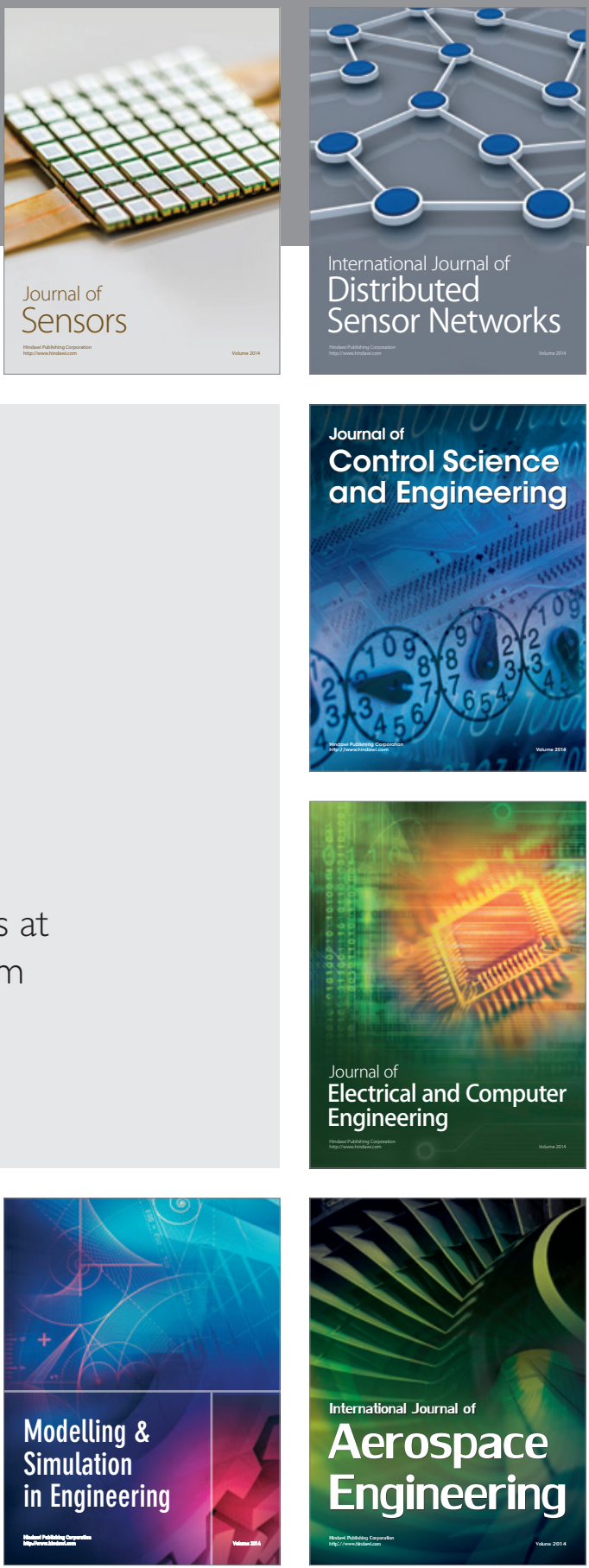

Journal of

Control Science

and Engineering
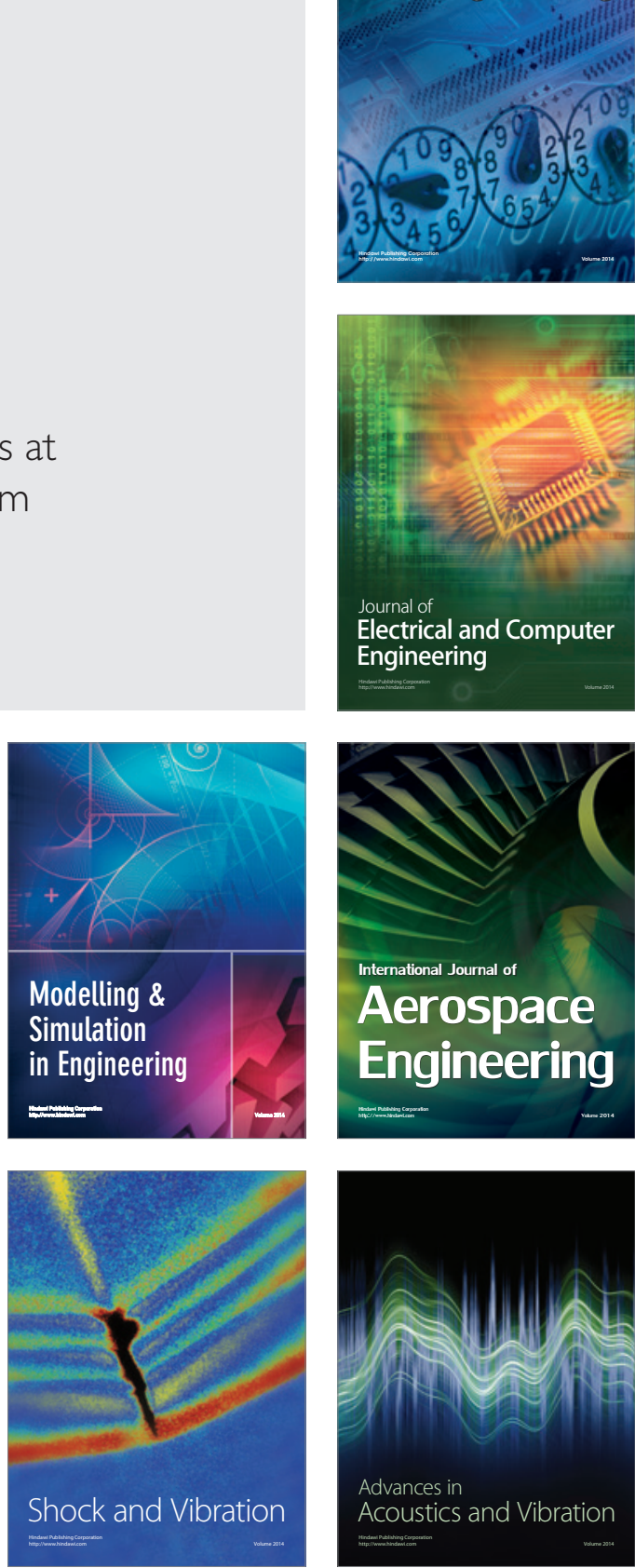